\title{
A Deformation Theory of Self-Dual Einstein Spaces
}

\author{
C. G. TORRE
}

\begin{abstract}
The self-dual Einstein equations on a compact Riemannian 4manifold can be expressed as a quadratic condition on the curvature of an $S U(2)$ (spin) connection which is a covariant generalization of the self-dual Yang-Mills equations. Local properties of the moduli space of self-dual Einstein connections are described in the context of an elliptic complex which arises in the linearization of the quadratic equations on the $S U(2)$ curvature. In particular, it is shown that the moduli space is discrete when the cosmological constant is positive; when the cosmological constant is negative the moduli space can be a manifold the dimension of which is controlled by the Atiyah-Singer index theorem.
\end{abstract}

\section{Introduction}

The last few years have seen remarkable progress in the theory of differentiable manifolds in 3 and 4 dimensions [1]. What is more remarkable, at least from a physicist's point of view, is the strong link these mathematical ideas have had with elements of field theory. In particular, in Donaldson's theory of 4-manifolds the moduli space of (gauge-inequivalent) solutions to the self-dual Yang-Mills equations plays the central role. This moduli space was originally studied by physicists (and mathematicians too) in the context of instanton contributions to functional integrals in quantum gauge theory, and recently it was shown by Witten [2] how in fact a quantum field theory- "topological YangMills theory" - provides a (necessarily somewhat heuristic) explanation for the success of Donaldson's approach.

If Yang-Mills theory, which is firmly rooted in particle physics, should have such a profound role to play in describing the global structure of 4-manifolds, one is naturally led to ask: What then is the role (if any) for the earliest of the modern geometrical theories, namely, general relativity? The answer to this

Work supported in part by NSF Grant PHY-9005790 to Syracuse University.

1991 Mathematics Subject Classification. Primary 53C25, 83C05.

This paper is in final form and no version will be submitted for publication elsewhere.

(C) 1992 American Mathematical Society 0271-4132/92 $\$ 1.00+\$ .25$ per page 
question may lie in another remarkable recent series of results that, roughly speaking, reveal a new way in which one can view gravitation as a gauge theory. What I have in mind here is the program initiated by Ashtekar [3] of viewing the dynamics of the gravitational field in terms of the left (or right) handed (equivalently: self-dual or anti-self-dual) spin connection. The new perspectives afforded by the ensuing "connection dynamics" point of view in general relativity have stimulated renewed interest and fresh results in the program of canonical quantization of the gravitational field.

As we shall see, the left-handed spin connection is also a useful variable for studying certain aspects of classical differential geometry, in particular, the geometry and topology of self-dual Einstein spaces. This was realized early on by Ashtekar, Jacobson, and Smolin [4] in their study of the $3+1$ form of half-flat solutions to the Einstein equations (with vanishing cosmological constant), and later it was shown by Capovilla, Dell, and Jacobson [5] that the self-dual Einstein equations (with non-vanishing cosmological constant) can be expressed purely in terms of the left-handed spin connection in a way which can be thought of as a covariant generalization of the self-dual Yang-Mills equations.

The work that we shall present here can be viewed as a first attempt to assess the feasibility of applying ideas from topological field theory to the moduli space of self-dual gravitational instantons. Because topological quantum field theories are nearly "classical" - typically the semi-classical approximation is exact-the majority of the topological field theory formalism is dominated by the features of the linearized theory, i.e., the deformation theory of the particular moduli space under consideration. Thus in this talk we aim to develop the deformation theory of self-dual Einstein spaces using the self-dual spin connection as the basic variable. We shall see that the techniques which were brought to bear in the corresponding Yang-Mills problem [6] can be fruitfully applied also in the gravitational case. At the very least, it will become clear that the direct use of the left-handed spin connection leads to new results in the theory of Einstein spaces at relatively little cost in the way of extensive computations.

The mathematical setting for what follows is an $S U(2)$ principal bundle with connection over a "spacetime", which will be taken to be a compact, smooth, Riemannian, 4-dimensional spin manifold $\mathbf{M}$, and associated vector bundles equipped with covariant derivatives. In this framework, left-handed spinors arise as sections of the vector bundle associated with the defining representation of $S U(2)$. We will primarily be concerned with the vector bundle constructed via the adjoint representation; the space of smooth sections of this bundle will be denoted $S_{0}$ and can be viewed as the symmetric tensor product of the bundle of left-handed spinors with itself. Given a soldering form, $S_{0}$ can be identified with the bundle of self-dual 2-forms. The tensor product of $S_{0}$ with the bundle of p-forms is denoted $S_{p}$. We will use Penrose's abstract index notation [13] to describe the various geometric objects under consideration. In particular, lowercase Latin indices will denote tensors on $\mathbf{M}$, and uppercase Latin indices 
denote $S U(2)$ spinors, i.e., sections of the various vector bundles. Spinor indices are lowered and raised with the $S U(2)$-invariant symplectic form $\epsilon_{A B}$ and its inverse $\epsilon^{A B}$. When dealing with elements of $S_{p}$ it will be convenient at times to use a matrix notation in which the spinor indices are suppressed; in this context square brackets [, ] will represent commutators in the Lie algebra $s u(2)$.

The emphasis of this presentation will be on developing formalism and presenting key results; no attempt will be made to be rigorous, e.g., with respect to functional analysis.

\section{Definition of the moduli space}

The self-dual Einstein equations are

$$
\begin{gathered}
R_{a b}=\Lambda g_{a b} \\
C_{a b c d}=-\frac{1}{2} \epsilon_{c d}{ }^{m n} C_{a b m n}
\end{gathered}
$$

where $R_{a b}, C_{a b c d}$, and $\epsilon_{a b c d}$ are the Ricci tensor, Weyl tensor, and volume form of the metric $g_{a b}$ respectively. $\Lambda$ is the "cosmological constant". One might, more accurately, call metrics satisfying (2) "conformally anti-self-dual", but for brevity we will simply refer to them as "self-dual".

When $\Lambda \neq 0$, eqs. (1) and (2) can be written in terms of an $S U(2)$ (spin) connection as follows. We first rewrite (1) in terms of a soldering form $\gamma_{a}^{A A^{\prime}}$, which is an isomorphism between vector fields and $S U(2) \times S U(2)$ spinors, and the self-dual part of the associated spin connection $\nabla_{a}: S_{p} \rightarrow S_{p+1}$. The spacetime metric is obtained via

$$
g_{a b}=\gamma_{a}^{A A^{\prime}} \gamma_{b A A^{\prime}},
$$

while the curvature of the left-handed spin connection is given by

$$
2 \nabla_{[a} \nabla_{b]} \alpha_{A}=F_{a b A}^{B} \alpha_{B},
$$

where $F_{a b}^{A B}=F_{a b}^{(A B)}$. If we define the self-dual 2-forms

$$
\Sigma_{a b}^{A B}:=2 \gamma_{[a}^{A A^{\prime}} \gamma_{b] A^{\prime}}^{B}
$$

which define the isomorphism between $S_{0}$ and the bundle of self-dual 2-forms mentioned above, $F_{a b}$ is related to the self-dual part of the Riemann tensor, $R_{a b c d}^{(+)}$, via

$$
R_{a b c d}^{(+)}=-\frac{1}{2} F_{a b}^{A B} \Sigma_{c d A B},
$$

or, in an $s u(2)$ matrix notation,

$$
R_{a b c d}^{(+)}=\frac{1}{2} t r F_{a b} \Sigma_{c d} .
$$


The Einstein equations (1) are equivalent to [7]

$$
\nabla_{[a} \Sigma_{b c]}^{A B}=0
$$

and

$$
\gamma_{[a}^{A A^{\prime}} F_{b c] A}^{B}+\frac{1}{6} \Lambda \gamma_{[a}^{A A^{\prime}} \Sigma_{b c] A}^{B}=0 .
$$

Eq. (8) enforces the condition that $\nabla_{a}$ is the covariant derivative coming from the left-handed (self-dual) part of the spin connection compatible with $\gamma_{a}$; given (8), (9) is equivalent to (1).

While the Einstein equations can be formulated in terms of a soldering form and the left-handed spin connection, it is rather remarkable that the self-dual Einstein equations can be written purely in terms of the spin connection via

$$
F_{[a b}^{(A B} F_{c d]}^{C D)}=0
$$

and

$$
\operatorname{det} \Phi>0,
$$

where $\Phi$ is a linear map from the space of symmetric rank-two spinors to symmetric rank-two spinor densities of weight one defined by

$$
\Phi_{C D}^{A B}:=\eta^{a b c d} F_{a b}^{A B} F_{c d C D} .
$$

Here $\eta^{a b c d}=\eta^{[a b c d]}$ is the Levi-Civita tensor density of weight one.

The relationship between $(10),(11)$ and $(1),(2)$ is as follows $[\mathbf{5 , 7}]$. The general solution to $(10)$ is

$$
F_{a b}^{A B}=-\frac{1}{3} \Lambda \gamma_{[a}^{A A^{\prime}} \gamma_{b] A^{\prime}}^{B}=-\frac{1}{6} \Lambda \Sigma_{a b}^{A B}
$$

where $\Lambda$, a constant with dimensions (length $)^{-2}$, is needed for dimensional reasons. If we interpret $\gamma_{a}^{A A^{\prime}}$ as a soldering form, the inequality (11) guarantees that the metric (3) is positive definite. Now, (13) solves (9) directly, and (8) is satisfied by virtue of the Bianchi identity:

$$
\nabla_{[a} F_{b c]}=0 \text {. }
$$

So, (10),(11) lead to a solution of the Einstein equations. As the 2-forms $\Sigma_{a b}$ are self-dual with respect to the metric which they define, so too is the $S U(2)$ field strength; it can be shown that the solutions generated in this manned have anti-self-dual Weyl tensor. Conversely, all anti-self-dual Einstein spaces (with $\Lambda \neq 0$ ) arise as solutions to (10),(11) [8]. Conformally self-dual (as opposed to anti-self-dual) Einstein spaces can be obtained by a change of orientation, which amounts to using the right-handed spin connection ("primed spinors")

Given a solution to (10),(11), we can generate infinitely many others by using the induced action of the automorphism group of the $S U(2)$ bundle being used ${ }^{1}$.

\footnotetext{
${ }^{1}$ I thank A. Fischer for patiently explaining to me the structure of this group.
} 
This group, which we shall loosely call the "gauge group", includes the usual local $S U(2)$ gauge group (familiar from Yang-Mills theory) as a normal subgroup; the diffeomorphism group of $\mathbf{M}$ appears as the factor group (via bundle projection). The space of gauge-inequivalent solutions to (10),(11), which we shall denote $\mathcal{M}$, is the natural "moduli space" of the problem. Our goal in what follows is to uncover some local properties of this moduli space by studying its tangent space $T \mathcal{M}$.

\section{Remarks}

The translation of the self-dual Einstein equations into a quadratic condition on the curvature of an $S U(2)$ connection is the result of a sequence of observations. Ashtekar and Renteln [4] noticed that, in the context of the $3+1$ formalism for (complex) general relativity in terms of Ashtekar's "new variables", all constraints are satisfied by the ansatz

$$
B^{a}=-\frac{1}{3} \Lambda E^{a}
$$

where $E^{a}$ is the densitized dual of the pull-back of $\Sigma_{a b}$ to a 3-dimensional submanifold of $\mathbf{M}$, and $B^{a}$ is the non-Abelian magnetic field: the densitized dual of the pull-back of $F_{a b}$. They also pointed out that the evolution of such initial data sets leads to self-dual Einstein spaces. It was Samuel [7] who gave the 4-dimensional version (13) of the ansatz and showed how it yields solutions of the Einstein equations; shortly thereafter it was shown that the ansatz was equivalent to the self-dual Einstein equations [8]. Capovilla, Dell and Jacobson [5] pointed out that all reference to the soldering form could be eliminated via (10).

Because the self-dual Einstein equations can be expressed in terms of an $S U(2)$ connection, it is easy to see that they are related to the self-dual Yang-Mills equations. Indeed, if we identify $-\frac{1}{3} \Lambda E^{a}$ in (15) with the Yang-Mills electric field, then (15) is precisely the pull-back of the Yang-Mills self-duality ansatz to a 3dimensional submanifold. Furthermore, because the field strength satisfying (13) is self-dual with respect to the metric it defines, all solutions to (10),(11) are also solutions of the self-dual Yang-Mills equations ${ }^{2}$ (although, of course, the converse is not true). One can think of (10) as a diffeomorphism covariant generalization of the self-dual Yang-Mills equations, the latter being non-covariant because of the need for an externally prescribed metric in their definition.

Note that there can, in principle, be topological obstructions to the existence of solutions to (10),(11) or (1),(2). For example, it is well-known that $\mathbf{M}$ must have vanishing first homology if it is to admit Einstein metrics with $\Lambda>0$. It is also easy to show that the Euler number and Hirzebruch signature of $\mathbf{M}$ must be

\footnotetext{
${ }^{2}$ For example, Samuel showed [7] that the spin connection of De Sitter space can be identified with the single instanton configuration in Yang-Mills theory.
} 
positive and negative semi-definite respectively. In what follows we will indicate how some new obstructions might arise.

\section{Deformation theory}

Let us now turn to the formal construction of the tangent space $T \mathcal{M}$ to the moduli space discussed above. Consider a 1-parameter family of solutions to (10),(11). A perturbation is a tangent vector to this curve at the point representing a given solution and is represented by an element $C$ of $S_{1}$,

$$
C_{a}^{A B}=C_{a}^{(A B)},
$$

satisfying

$$
F_{[a b}^{(A B} \nabla_{c} C_{d]}^{C D)}=0
$$

where $\nabla_{a}$ and $F_{a b}$ are built from the unperturbed solution. The infinite-dimensional vector space of solutions to (17) can be projected to $T \mathcal{M}$ by identifying any two perturbations $C_{a}$ and $C_{a}^{\prime}$ which differ by an infinitesimal gauge transformation, i.e.,

$$
C_{a} \sim C_{a}^{\prime}
$$

if

$$
C_{a}-C_{a}^{\prime}=N^{b} F_{b a}+\nabla_{a} N
$$

where $N^{a}$ is a (complete) vector field associated with an infinitesimal diffeomorphism of $\mathbf{M}$ and $N \in S_{0}$ generates an infinitesimal $S U(2)$ gauge transformation. It is straightforward to verify that any "pure gauge" perturbation (18) satisfies the linearized equations (17).

The form (18) of infinitesimal automorphisms arises as follows. A 1-parameter family of automorphisms yields a complete vector field on the $S U(2)$ principal bundle where the connection naturally lives as an $s u(2)$-valued 1-form; the "infinitesimal" action of the automorphism group on the connection is the Lie derivative of the connection 1 -form along this vector field, which can be identified with an element of $S_{1}$. Using the fixed unperturbed connection, the vector field can be split into horizontal and vertical parts; the horizontal part yields the first term in (18), which can be thought of as a "gauge covariant Lie derivative", while the vertical part of the vector field generating the automorphism leads to the second term in (18) in the familiar way.

Crucial for the results to follow is that when $F_{a b}$ satisfies (10),(11), and hence (13), the r.h.s of (18) can be written as

$$
N^{b} F_{b a}+\nabla_{a} N=\left(\nabla^{b} f\right) F_{b a}+\left[\nabla^{b} L, F_{b a}\right]+h^{b} F_{b a}+\nabla_{a} \hat{N},
$$

where $f \in C^{\infty}(M)$ is a real-valued function, $L, \hat{N}$ are elements of $S_{0}$ and the background self-dual Einstein metric is used to provide the isomorphism between vector fields and 1-forms. Eq. (19) results from a Hodge decomposition of $N_{a}$ : 
$\nabla_{a} f$ is the exact part of $N_{a}, L$ comes from the co-exact part, and $h_{a}$ is the harmonic part of $N_{a}$. For details, see [9]. To simplify the results which follow, let us henceforth assume that $\mathbf{M}$ has vanishing first homology so that $h_{a}$ is in fact zero $^{3}$.

The tangent space to moduli space (where it is well-defined) can now be characterized as follows. Let $W_{4}$ denote the space of smooth sections constructed as the product of the bundle of 4 -forms with the totally symmetric trace-free tensor product of $S_{0}$ with itself, e.g., if $\omega_{a b c d}^{A B C D} \in W_{4}$, then

$$
\omega_{a b c d}^{A B C D}=\omega_{a b c d}^{(A B C D)} .
$$

Define the following linear differential operators:

$$
\begin{gathered}
D_{0}: C^{\infty}(M) \oplus S_{0} \oplus S_{0} \rightarrow S_{1}, \\
D_{0}(f, L, N):=\left(\nabla^{b} f\right) F_{b a}+\left[\nabla^{b} L, F_{b a}\right]+\nabla_{a} N
\end{gathered}
$$

and

$$
\begin{gathered}
D_{1}: S_{1} \rightarrow W_{4}, \\
D_{1} C=F_{[a b}^{(A B} \nabla_{c} C_{d]}^{C D)} .
\end{gathered}
$$

Because any perturbation which is "pure gauge" satisfies the linearized equations, we have

$$
D_{1} D_{0}=0 \text {. }
$$

The tangent space $T \mathcal{M}$ to moduli space, at a given point representing the unperturbed self-dual Einstein space, is then simply

$$
T \mathcal{M}=\frac{\operatorname{Ker} D_{1}}{\operatorname{Im} D_{0}} .
$$

We have thus arrived at a cohomological description of $T \mathcal{M}$. In particular, the differential complex

$$
C^{\infty}(M) \oplus S_{0} \oplus S_{0} \stackrel{D_{0}}{\longrightarrow} S_{1} \stackrel{D_{1}}{\longrightarrow} W_{4}
$$

is elliptic, i.e., the symbol sequence is exact, so exactly as in Hodge theory we can characterize $T \mathcal{M}$ by the kernel of an elliptic differential operator. To do this we need inner products on the various sections which feature in the complex. The inner products are constructed using the unperturbed self-dual Einstein metric and the $s u(2)$ trace (equivalently: the symplectic form $\epsilon_{A B}$ ), e.g., for $C, C^{\prime} \in S_{1}$ we set

$$
\left(C^{\prime}, C\right):=-\int_{M} \sqrt{g} g^{a b} \operatorname{tr} C_{a}^{\prime} C_{b}
$$

\footnotetext{
${ }^{3}$ Vanishing first homology is guaranteed, e.g., if $\mathbf{M}$ is simply connected.
} 
with obvious generalizations to sections of the other bundles. Using this inner product one obtains the following adjoint operators

$$
\begin{gathered}
D_{0}^{*}: S_{1} \rightarrow C^{\infty}(M) \oplus S_{0} \oplus S_{0} \\
D_{0}^{*} C=\left(t r F^{a b} \nabla_{a} C_{b} ;\left[\nabla_{a} C_{b}, F^{a b}\right] ;-\nabla^{a} C_{a}\right), \\
D_{1}^{*}: W_{4} \rightarrow S_{1} \\
D_{1}^{*} \omega=F_{C D}^{c d} \nabla^{b} \omega_{a b c d}^{A B C D},
\end{gathered}
$$

and "Laplacians"

$$
\Delta_{0}: C^{\infty}(M) \oplus S_{0} \oplus S_{0} \rightarrow C^{\infty}(M) \oplus S_{0} \oplus S_{0}
$$

$$
\begin{gathered}
\Delta_{0}=D_{0}^{*} D_{0}, \\
\Delta_{1}: S_{1} \rightarrow S_{1} \\
\Delta_{1}=D_{1}^{*} D_{1}+D_{0} D_{0}^{*}, \\
\Delta_{2}: W_{4} \rightarrow W_{4} \\
\Delta_{2}=D_{1} D_{1}^{*},
\end{gathered}
$$

which are elliptic second-order partial differential operators. In (27),(28) we have extended the action of $\nabla_{a}$ to include tensors via the connection compatible with the background self-dual Einstein metric.

The Fredholm alternative implies the orthogonal decomposition

$$
S_{1}=\operatorname{Ran} D_{0} \oplus \operatorname{Ran} D_{1}^{*} \oplus \operatorname{Ker} \Delta_{1},
$$

from which it is easy to show that

$$
\frac{\operatorname{Ker} D_{1}}{\operatorname{Im} D_{0}}=\operatorname{Ker} \Delta_{1}=\operatorname{Ker} D_{1} \cap \operatorname{Ker} D_{0}^{*} .
$$

Because $\Delta_{1}$ is elliptic-hence Fredholm-we see that $\mathcal{M}$ is finite-dimensional.

Just as the alternating sum of the dimension of the kernels of Laplacians in Hodge theory defines a topological invariant (the Euler number), the alternating sum of the dimension of the kernels of the above Laplacians is a topological invariant. Let $I$ denote the "topological index" associated with the complex (25) [6]. It is completely determined by the topology of $\mathbf{M}$ and the $S U(2)$ bundle over $\mathbf{M}$. The Atiyah-Singer index theorem then implies

$$
I=\operatorname{dim} K e r \Delta_{0}-\operatorname{dim} K e r \Delta_{1}+\operatorname{dimKer} \Delta_{2},
$$

so, provided $\operatorname{Ker} \Delta_{0}=0=\operatorname{Ker} \Delta_{2}$ (see the next section), the dimension of moduli space (if it exists and is a manifold) is determined by the topology of $\mathbf{M}$ via the index $I$. 


\section{Remarks}

I do not yet have an explicit expression for $I$; it is evidently a linear combination of the Chern number $k$ of the $S U(2)$ bundle being used as well as the Euler number $\chi$ and modulus of the signature $|\tau|$, which are both invariants ${ }^{4}$ of $\mathbf{M}$. Because the $S U(2)$ bundle describes spinors on $\mathbf{M}$, it is possible to relate the second Chern number to $\chi$ and $\tau$ (see (6)):

$$
k=\frac{1}{2} \chi+\frac{3}{4} \tau
$$

so $I$ can be expressed as a linear combination of $\chi$ and $|\tau|$. Notice that, because $k$ must be an integer and $\tau$ is a multiple of 8 for a spin manifold, $\chi$ must be an even integer.

For some purposes it is useful to rearrange the elliptic complex as follows. Let

$$
D: S_{1} \rightarrow C^{\infty}(M) \oplus S_{0} \oplus S_{0} \oplus W_{4}
$$

$$
D:=D_{0}^{*} \oplus D_{1}
$$

$$
D^{*}: C^{\infty}(M) \oplus S_{0} \oplus S_{0} \oplus W_{4} \rightarrow S_{1}
$$

$$
D^{*}:=D_{0} \oplus D_{1}^{*}
$$

$D$ is an elliptic operator; the Fredholm alternative implies the orthogonal decomposition

$$
S_{1}=\operatorname{Ran} D^{*} \oplus \operatorname{Ker} D
$$

and we have

$$
T \mathcal{M}=\operatorname{Ker} D
$$

The Atiyah-Singer index theorem now reads

$$
I=\operatorname{dim} K e r D^{*}-\operatorname{dim} K e r D .
$$

A similar elliptic complex arises in the deformation theory of the moduli space of self-dual Yang-Mills connections. The first space in (25) is replaced by $S_{0}$ (representing infinitesimal $S U(2)$ gauge transformations, i.e., vertical automorphisms), the second space of sections is again $S_{1}$, while the third space is the product of $S_{0}$ and the space of anti-self-dual 2-forms. The topological index in this case is again determined by $k, \chi, \tau$, which are all independent in this case.

\footnotetext{
${ }^{4}$ Only the absolute value of $\tau$ can appear because the dimension of the kernels of the Laplacians does not depend on the orientation of $\mathbf{M}$.
} 


\section{Vanishing theorems, linearization stability}

It turns out that the properties of the moduli space depend rather strongly on the sign of the cosmological constant $\Lambda$. To see this, we shall show that, when $\Lambda>0, \operatorname{Ker} \Delta_{1}=0=\operatorname{Ker} \Delta_{2}$, while $\operatorname{Ker} \Delta_{0}=0$ when $\Lambda<0$, and explore the consequences of these "vanishing theorems".

Let us begin with $\operatorname{Ker} \Delta_{1}$, which formally defines the tangent space to moduli space. We have seen that $T \mathcal{M}=\operatorname{Ker} \Delta_{1}=\operatorname{Ker} D_{1} \cap \operatorname{Ker} D_{0}^{*}$, which can be understood as expressing $T \mathcal{M}$ as the space of self-dual perturbations $\left(\operatorname{Ker} D_{1}\right)$ in a particular gauge $\left(\operatorname{Ker} D_{0}^{*}\right)$. Given that $C \in \operatorname{Ker} D_{0}^{*}$ we have

$$
\Delta_{1} C=D_{1}^{*} D_{1} C
$$

where

$$
D_{1} C=\frac{1}{12} \epsilon_{a b c d} F^{m n C D} \nabla_{m} C_{n}^{A B}
$$

(note: in (42) we have used $D_{0}^{*} C=0$ to remove the symmetrization on spinor indices; we have also used the self-duality of $F_{a b}$ ). Explicit computation then reveals

$$
\Delta_{1} C=\frac{\Lambda^{2}}{54} \nabla^{b}\left[\left(\delta_{a}^{[c} \delta_{b}^{d]}+\frac{1}{2} \epsilon_{a b}^{c d}\right) \nabla_{c} C_{d}\right]
$$

where we have again extended the action of $\nabla_{a}$ to tensors via the metric compatible connection of the background geometry. Using $D_{0}^{*} C=0 \Longrightarrow \nabla^{a} C_{a}=0$, it follows from (43) that

$$
C \in \operatorname{Ker} \Delta_{1} \Longrightarrow\left(-\nabla^{a} \nabla_{a}+\Lambda\right) C_{b}=0
$$

which is a remarkably simple elliptic partial differential equation for $C_{b}$. It is easily seen that the linear operator $-\nabla^{a} \nabla_{a}+\Lambda$ is positive definite when $\Lambda>0$ (relative to the inner-product introduced above) and hence we see that $\operatorname{Ker} \Delta_{1}=0$ when $\Lambda>0$. This means that, when the cosmological constant is positive, all perturbations of self-dual connections are "pure gauge", i.e., all instantons are isolated - the moduli space is discrete.

A straightforward computation reveals that

$$
\Delta_{2} \omega=0 \Longleftrightarrow\left(-\nabla^{a} \nabla_{a}+2 \Lambda\right) \omega^{A B C D}=0,
$$

where

$$
\omega^{A B C D}:=\epsilon^{a b c d} \omega_{a b c d}^{A B C D} .
$$

Again, we see that $\Delta_{2}$ is a positive definite operator and $\operatorname{Ker} \Delta_{2}=0$ when $\Lambda>0$. Finally, in a similar fashion, it can be shown [10] that $\operatorname{Ker} \Delta_{0}=0$ when $\Lambda<0$. In light of these vanishing theorems, the Atiyah-Singer index theorem yields

$$
\begin{aligned}
& \Lambda>0: \quad I=\operatorname{dimKer} \Delta_{0}, \\
& \Lambda<0: \quad I=\operatorname{dimKer} \Delta_{2}-\operatorname{dimKer} \Delta_{1} .
\end{aligned}
$$


So, when $\Lambda>0, \mathcal{M}$ is zero-dimensional and the dimension of $\operatorname{Ker} \Delta_{0}$ is controlled by the topology of M. A closer look at $\operatorname{Ker} \Delta_{0}$ reveals [10] that this space can be identified with the space of Killing vectors of the unperturbed self-dual Einstein metric. Thus the dimension of the isometry group of a given self-dual Einstein metric is controlled by the Euler number and signature of $\mathbf{M}$.

When $\Lambda<0$ the dimension of moduli space is also controlled by $\chi$ and $|\tau|$ provided that $\operatorname{Ker} \Delta_{2}=0$. When $\operatorname{Ker} \Delta_{2} \neq 0$ one must confront the issue of linearization stability: it is possible that some solutions to (17) do not come from a 1-parameter family of solutions to (10),(11). Because solutions to $\Delta_{1} C=0$ are to represent tangent vectors to moduli space, spurious solutions arise when $\mathcal{M}$ has singular points where the tangent space is not well-defined. Using the implicit function theorem it is easy to see that a non-trivial kernel for $\Delta_{2}$ allows for an obstruction to the existence of a manifold structure for $\mathcal{M}$. More precisely, the implicit function theorem implies that $\mathcal{M}$ exists as a manifold in the neighborhood of a self-dual instanton provided $D$ is surjective. From the splitting

$$
C^{\infty}(M) \oplus S_{0} \oplus S_{0} \oplus W_{4}=\operatorname{Ran} D \oplus K e r D^{*}
$$

it is clear that $D$ is surjective provided $\operatorname{Ker} D^{*}=0$, but, when $\Lambda<0, \operatorname{Ker} D^{*}=$ $\operatorname{Ker} \Delta_{2}$. So, provided $\operatorname{Ker} \Delta_{2}=0$, the equations (10),(11) are linearization stable by virtue of the fact that $\mathcal{M}$ exists (locally) as an $I$-dimensional submanifold of the space of all $S U(2)$ connections. Otherwise, one has to contend with the possible appearance of singularities in $\mathcal{M}$ where, strictly speaking, first-order perturbation theory fails.

\section{Remarks}

Typically, the singularities which occur in a moduli problem come from quotienting by the action of a symmetry (gauge) group which has fixed points. This is not the case here. Indeed, when $\Lambda<0, \operatorname{Ker} \Delta_{0}=\operatorname{Ker} D_{0}=0$ so there are no fixed points. The singularities which can occur stem from pathological behavior of the self-duality equations $(10),(11)$. To see this note that the Fredholm alternative implies the orthogonal decomposition

$$
W_{4}=\operatorname{Ran} D_{1} \oplus \operatorname{Ker} D_{1}^{*},
$$

so $D_{1}$ is surjective only if $\operatorname{Ker} D_{1}^{*}=\operatorname{Ker} \Delta_{2}=0$. Hence the singularities (if any) are already present once one restricts to the infinite-dimensional subspace (10),(11).

It is interesting to note that the sign of the topological index can represent an obstruction to the existence of a self-dual Einstein metric (or connection) with a given sign for the cosmological constant. If $I<0$ then clearly (47) cannot be satisfied when $\Lambda>0$. Similarly, if the topology of $\mathbf{M}$ is such that $I>0$, 
then there can be no self-dual Einstein spaces with $\Lambda<0$ (away from points of linearization instability).

In the deformation theory of self-dual Yang-Mills connections one can also prove certain vanishing theorems which are relevant to linearization stability as well as to the existence of symmetries associated with reducible connections. It is also possible to have a discrete moduli space, but this depends on the topology of the base manifold and $S U(2)$ bundle. Singularities can appear in the Yang-Mills version of $\mathcal{M}$; they arise from the above mentioned symmetries (which represent fixed points for the action of the gauge group) and/or from the failure of the self-dual Yang-Mills equations to define a sub-manifold.

\section{Concluding remarks}

By studying the local properties of the space of solutions to the self-dual Einstein equations we have seen the strong interplay between self-dual geometry and the topology of 4-manifolds. It is now time to assess how far we have come toward implementing the goals expressed in the introduction, i.e., we should now ask: can the moduli space of self-dual Einstein connections tell us anything about the topology of 4-manifolds? First of all, it is clear that while studying the deformation theory of $\mathcal{M}$ is certainly necessary for answering this question, it is far from sufficient. What is needed to construct a gravitational analog of Donaldson theory is to gain control over the behavior of $\mathcal{M}$ in the large. It is of course going to be a non-trivial problem to get an analogous level of understanding of the gravitational moduli space when $\Lambda<0$ as one has for the moduli space of self-dual Yang-Mills connections. On the other hand, for manifolds admitting gravitational instantons with $\Lambda>0$, the moduli space is discrete and one already knows in the Yang-Mills case that a discrete moduli space leads to a new invariant for smooth 4-manifolds.

From a physicist's point of view, the "explanation" of the success of Donaldson theory was given by Witten via topological Yang-Mills theory. It is therefore encouraging to note that many features of Witten's topological Yang-Mills theory can be reproduced in the gravitational case. Indeed, as shown in [11] the classical aspects of the construction of Witten's theory have a natural diffeomorphism invariant generalization to the gravitational case. The use of an $S U(2)$ connection to describe both gauge and gravitational instantons leads to strong similarities between both topological field theories and one can hope that detailed analysis will lead to a similar degree of success in the gravitational case as was obtained via Yang-Mills theory. What is needed to complete the work of [11] is a better understanding of the quantum functional measure: the existence of a measure on connections which is consistent with the inequality (11) is bound to be a highly non-trivial issue. Alternatively (equivalently?), the theory may be profitably developed using the Hamiltonian formalism and canonical quantization. In addition, one needs a better understanding of the singularity structure 
of $\mathcal{M}$, which by the way is also a difficult issue in topological Yang-Mills theory.

We have from time to time compared the deformation theory of the space of gravitational instantons to the corresponding deformation theory of Yang-Mills instantons. It is possible to give another analogy which also serves to summarize the key results of the work presented here, namely, I would like to argue that the moduli space of self-dual Einstein connections is a natural 4-dimensional generalization of the moduli space of Riemann surfaces. Every metric on a compact Riemannian 2-manifold is conformal to an Einstein metric, where $\Lambda>0$ for genus 0 , the sphere, $\Lambda=0$ for genus 1 , the torus, and $\Lambda<0$ for genus $\geq 2$. In both the gravitational and Riemann surface cases the moduli space is discrete when $\Lambda>0$ and the dimension of the isometry group of the Einstein metric is controlled by the Atiyah-Singer index theorem (which becomes the RiemannRoch theorem in two dimensions). When $\Lambda<0$ the moduli space appears to be a manifold in each case whose dimension is again controlled by the topology of the two or four dimensional manifold via the index theorem. (The $\Lambda=0$ case, which, it seems, cannot be handled via (10), is well known-it is the K3 geometry, which can be thought of as the 4-dimensional generalization of the torus in Riemann surface theory.) The analogy between self-dual Einstein connections in 4 dimensions and Riemann surface theory in 2 dimensions is further strengthened by the observation that the moduli space of Riemann surfaces is identifiable with the diffeomorphism equivalence classes of complex structures on the compact 2manifold. Similarly, it is easy to see that the moduli space of self-dual Einstein connections is closely related to the space of quaternionic Kähler structures on a compact Riemannian 4-manifold [12]. More precisely, let $\sigma_{\underline{i}}, \underline{i}=1,2,3$ denote a basis in $s u(2)$, then by solving (10) and (11) one can construct three almost complex structures,

$$
J_{\underline{i} a}{ }^{b}:=-\left(\frac{3 \sqrt{2}}{\Lambda}\right) \operatorname{tr} \sigma_{\underline{i}} F_{a}{ }^{b},
$$

satisfying the algebra of quaternions.

I would like to thank A. Ashtekar and J. Samuel for discussions.

\section{REFERENCES}

1. See, e.g., the review of M. Atiyah, Quantum field theory and low-dimensional geometry, Prog. Th. Phys. Suppl. 102 (1990), 1-13.

2. E. Witten, Topological quantum field theory, Commun. Math. Phys. 117 (1988), 353-386.

3a. A. Ashtekar, New Hamiltonian formulation of general relativity, Phys. Rev. D 36 (1987), 1587-1602.

3b. _ New Perspectives in Canonical Gravity, Bibliopolis, Naples, 1988.

3c. —_ Lectures on Non-Perturbative Canonical Gravity, World Scientific, Singapore, 1991.

4a. A. Ashtekar, A 3+1 formulation of Einstein self-duality, Mathematics and General Relativity (J. Isenberg, ed.), American Mathematical Society, Providence, 1987, pp. 39-53. 
4b. A. Ashtekar, T. Jacobson, and L. Smolin, A new characterization of half-flat solutions to Einstein's equations, Commun. Math. Phys. 115 (1988), 631-648.

5. R. Capovilla, J. Dell, and T. Jacobson, Gravitational instantons as SU(2) gauge fields, Class. Quantum Grav. 7 (1990), L1-L3.

6. See, e.g., B. Boos and D. Bleecker, Topology and Analysis: The Atiyah-Singer Index Formula and Gauge Theoretic Physics, Springer-Verlag, New York, 1985.

7. J. Samuel, Gravitational instantons from the Ashtekar variables, Class. Quantum Grav. 5 (1988), L123-L125.

8. S. Koshti and N. Dadich, On the self-duality of the Weyl tensor using Ashtekar's variables, Class. Quantum Grav. 7 (1990), L5-L7.

9. C. G. Torre, Perturbations of gravitational instantons, Phys. Rev. D 41 (1990), 3620-3627.

10. - On the linearization stability of the conformally (anti-) self-dual Einstein equations, J. Math. Phys. 31 (1990), 2983-2986.

11. _ A topological field theory of gravitational instantons, Phys. Lett. B 252 (1990), 242-246.

12. D. Robinson, Half-flat solutions of Einstein's equations and hyper-Kähler structures, New Perspectives in Canonical Gravity, Bibliopolis, Naples, 1988, pp. 187-193.

13. R. Penrose and W. Rindler, Spinors and Spacetime, Vol. 1, Cambridge University Press, Cambridge, 1984.

Department of Physics, Syracuse University, Syracuse, New York 13244-1130

Department of Physics, Utah State University, Logan, Utah 84322-4415 